\title{
A CHARACTERIZATION OF FOURIER AND RADON TRANSFORMS ON EUCLIDEAN SPACE \\ BY
}

\author{
ALEXANDER HERTLE
}

\begin{abstract}
We show that a continuous operator behaving under rotations, positive dilations, and translations like the Fourier or the Radon transform on $\mathbf{R}^{n}$ must be a constant multiple of one of these transforms. We prove this characterization for various function spaces, e.g. we characterize the Fourier transform as an operator acting on spaces between $\mathscr{D}\left(\mathbf{R}^{n}\right)$ and $\mathscr{D}^{\prime}\left(\mathbf{R}^{n}\right)$. On the other hand, a counterexample shows that the Radon transform is not determined by its behaviour above as an operator from $\mathscr{D}\left(\mathbf{R}^{n}\right)$ to $\mathscr{D}^{\prime}\left(S^{n-1} \times \mathbf{R}\right)$. But we can characterize the Radon transform as an operator acting between $\mathscr{D}\left(\mathbf{R}^{n}\right)$ and $\mathscr{D}_{L^{\prime}}^{\prime}\left(S^{n-1} \times \mathbf{R}\right)$, the space of integrable distributions on $S^{n-1} \times \mathbf{R}$. In the special case $n=1$, our methods sharpen results of J. L. B. Cooper and H. Kober, who characterize the Fourier transform as an operator from $L^{p}(\mathbf{R})$ into $L^{p^{\prime}}(\mathbf{R}), 1 \leqslant p \leqslant 2$.
\end{abstract}

Introduction. Several authors have been concerned with the question whether an operator on function spaces on $\mathbf{R}^{n}$ is determined up to a constant multiple by its behaviour under rotations, dilations, and translations. For instance, Stein [15] showed that the (translation invariant) Hilbert and Riesz transforms on $L^{2}$ can be characterized in such a way.

The Fourier and Radon transforms on $\mathbf{R}^{n}$ also have a well-known simple behaviour under rotations, positive dilations, and translations. In this article, we prove that such a behaviour is characteristic: An operator acting under those operations like the Fourier or the Radon transform is a constant multiple of one of these transforms.

In the case of the Fourier transform for $n=1$, our methods (e.g. determining homogeneous distributions by Euler's equation) sharpen results of Cooper [1] and Kober [10], who characterized by different methods the Fourier transform as an operator from $L^{p}(\mathbf{R})$ into $L^{p^{\prime}}(\mathbf{R}), 1 \leqslant p \leqslant 2$. We also obtain connections to Hardy [7] and Plancherel [12], who looked for operators on $L^{2}(\mathbf{R})$ behaving under dilations like the Fourier transform. Further, our characterizations can be used to obtain corresponding ones for operators related to the Fourier and Radon transforms (our result implies the result on Hilbert and Riesz transforms mentioned above, Stein [15]). We also remark that such characteristic properties can be applied to verify inversion formulas for the corresponding operator (in the case of the Radon

Received by the editors July 29, 1980 and, in revised form, May 12, 1981.

1980 Mathematics Subject Classification. Primary 42B10, 39B99, 44A15.

Key words and phrases. Fourier transform, Radon transform, functional equations.

(C)1982 American Mathematical Society 0002-9947/81/0000-0629/\$04.25 
transform see Gelfand, Graev, and Vilenkin $[5, \S 1.4])$. Finally, we discuss the possibility of more general characterizations and give some counterexamples.

In $\S 1$, we characterize the Fourier transform, which we denote by $F$. We are concerned with the spaces $\mathscr{D}\left(\mathbf{R}^{n}\right)$ of $C^{\infty}$ functions on $\mathbf{R}^{n}$ with compact support and $\mathscr{Z}\left(\mathbf{R}^{n}\right)$, the space of Fourier transforms of $\mathscr{D}$. We equip $\mathscr{D}$ and $\mathscr{Z}$ with their usual test function topologies. By $\mathscr{D}^{\prime}\left(\mathbf{R}^{n}\right)$ and $\mathscr{Z}^{\prime}\left(\mathbf{R}^{n}\right)$ we denote the duals of $\mathscr{D}$ and $\mathscr{Z}$, respectively, provided with their weak topology (cf. Gelfand and Shilov [4]). If $f$ is a function or a distribution on $\mathbf{R}^{n}$, then $f_{U}$ denotes its rotation by an orthogonal $U \in O(n), f_{\delta}$ its dilation by $\delta>0$, and $f_{a}$ its translation by $a \in \mathbf{R}^{n}$. Now the Fourier transform has the following behaviour:

$$
\begin{aligned}
& F\left(f_{U}\right)=(F f)_{U}, \\
& F\left(f_{\delta}\right)=\delta^{-n}(F f)_{1 / \delta}, \\
& F\left(f_{a}\right)=e^{i\langle a \cdot \cdot\rangle} F(f) .
\end{aligned}
$$

Conversely, we prove that any continuous operator $\tilde{F}$ from $\mathscr{D}\left(\mathbf{R}^{n}\right)$ or $\mathscr{Z}\left(\mathbf{R}^{n}\right)$ into $\mathscr{D}^{\prime}\left(\mathbf{R}^{n}\right)$ or $\mathscr{Z}^{\prime}\left(\mathbf{R}^{n}\right)$ satisfying (0.1)-(0.3) must be a constant multiple of the Fourier transform. Requiring the range of $\tilde{F}$ to be in $C\left(\mathbf{R}^{n}\right)$, we show that condition $(0.1)$ is superfluous.

In §2, we characterize the Radon transform, which we denote by $R$. The Radon transform is a continuous operator from $L^{1}\left(\mathbf{R}^{n}\right)$ into $L^{1}\left(S^{n-1} \times \mathbf{R}\right)$ and has the following behaviour:

$$
\begin{aligned}
& R\left(f_{U}\right)(\omega, p)=(R f)(U \omega, p), \\
& R\left(f_{\delta}\right)(\omega, p)=\delta^{1-n}(R f)(\omega, \delta p), \\
& R\left(f_{a}\right)(\omega, p)=(R f)(\omega, p+\langle\omega, a\rangle) .
\end{aligned}
$$

A counterexample shows that an operator $\tilde{R}$ from $\mathscr{D}\left(\mathbf{R}^{n}\right)$ into $\mathscr{D}^{\prime}\left(S^{n-1} \times \mathbf{R}\right)$ with $\left(0.1^{\prime}\right)-\left(0.3^{\prime}\right)$ is not determined up to a constant multiple. But restricting the range of $\tilde{R}$ to be a space of integrable distributions $\mathscr{D}_{L^{\prime}}^{\prime}$ (cf. Schwartz [13]), we prove (using tensor representations in the range) that any continuous operator $\tilde{R}$ acting between $\mathscr{D}\left(\mathbf{R}^{n}\right)$ and $\mathscr{D}_{L^{\prime}}^{\prime}\left(S^{n-1} \times \mathbf{R}\right)$ and satisfying $\left(0.1^{\prime}\right)-\left(0.3^{\prime}\right)$ is a constant multiple of the Radon transform. Finally we discuss the possibility to enlarge the range of $\tilde{R}$, applying results from $\$ 1$.

Throughout the paper, we shall use the notations of Gelfand and Shilov [4] and Schwartz [13]. Concerning the Radon transform, we use the notations of Gelfand, Graev, and Vilenkin [5] and Ludwig [11].

Special versions of Theorems 1 and 2 are contained in the author's thesis [8], supervised by Professor D. Kölzow. The author is also indebted to Professor B. Gramsch for helpful remarks and discussions.

1. A characterization of the Fourier transform. In this section, we prove that the Fourier transform is characterized as an operator from $\mathscr{D}\left(\mathbf{R}^{n}\right)$ into $\mathscr{D}^{\prime}\left(\mathbf{R}^{n}\right)$ by its behaviour with respect to rotations, positive dilations, and translations. 
We first fix our notations. Let $U \in O(n)$ denote a rotation in $\mathbf{R}^{n}, \delta>0$ a dilation factor, and $a \in \mathbf{R}^{n}$ a translation vector. If $f$ is a function on $\mathbf{R}^{n}$, we define the functions $f_{U}, f_{\delta}$, and $f_{a}$ by

$$
f_{U}(x)=f(U x), \quad f_{\delta}(x)=f(\delta x), \quad f_{a}(x)=f(x+a) .
$$

We extend these operations to distributions in the usual way. For $T \in \mathscr{Q}^{\prime}\left(\mathbf{R}^{n}\right)$ we define

$$
\left\langle T_{U}, \psi\right\rangle=\left\langle T, \psi_{U^{-1}}\right\rangle,\left\langle T_{\delta}, \psi\right\rangle=\delta^{-n}\left\langle T, \psi_{1 / \delta}\right\rangle, \quad\left\langle T_{a}, \psi\right\rangle=\left\langle T, \psi_{-a}\right\rangle .
$$

If $f \in L^{1}\left(\mathbf{R}^{n}\right)$, the Fourier transform of $f($ denoted by $F f$ or $\hat{f})$ is defined by

$$
(F f)(y)=\hat{f}(y)=(2 \pi)^{-n / 2} \int_{\mathbf{R}^{n}} e^{-i\langle x, y\rangle} f(x) d x .
$$

The Fourier transform of a distribution $T \in \mathscr{Q}^{\prime}\left(\mathbf{R}^{n}\right)$ is given as an element of $\mathscr{Z}^{\prime}\left(\mathbf{R}^{n}\right)$ by

$$
\langle\hat{T}, \hat{\psi}\rangle=\langle T, \tilde{\psi}\rangle, \quad \psi \in \mathscr{D}\left(\mathbf{R}^{n}\right) .
$$

We equip $\mathscr{D}, \mathscr{Z}$ with their usual topologies and $\mathscr{D}^{\prime}, \mathscr{Z}^{\prime}$ with their weak topologies. The Fourier transform is a continuous operator from $\mathscr{D}$ on $\mathscr{Z}$, and hence from $\mathscr{D}$ into $D^{\prime}$ (see Gelfand and Shilov [4]). Throughout the paper, by continuity of an operator we always mean sequential continuity.

It is well known that the Fourier transform has the properties $(0.1)-(0.3)$ in the introduction. Conversely, we can prove

THEOREM 1. Let $\tilde{F}: \mathscr{D}\left(\mathbf{R}^{n}\right) \rightarrow \mathscr{D}^{\prime}\left(\mathbf{R}^{n}\right)$ be a continuous operator. Then $\tilde{F}$ is a constant multiple of the Fourier transform, if and only if

(1) $\tilde{F}\left(\psi_{U}\right)=(\tilde{F} \psi)_{U}$,

(2) $\tilde{F}\left(\psi_{\delta}\right)=\delta^{-n}(\tilde{F} \psi)_{1 / \delta}$,

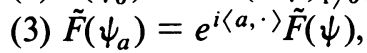

where $\psi \in \mathscr{D}\left(\mathbf{R}^{n}\right), U \in O(n), \delta>0$, and $a \in \mathbf{R}^{n}$.

Proof. Let $\tilde{F}$ : $\mathscr{Q}\left(\mathbf{R}^{n}\right) \rightarrow \mathscr{Q}^{\prime}\left(\mathbf{R}^{n}\right)$ be a continuous operator satisfying (1)-(3). We have to show that $\tilde{F}$ is a constant multiple of the Fourier transform.

To this purpose, let $\alpha, \psi \in \mathscr{D}\left(\mathbf{R}^{n}\right)$ and $\alpha$ radial with $\int \alpha(x) d x=1$ and $\alpha_{j}(x)=$ $j^{n} \alpha(j x), j \geqslant 1$, i.e. $\left(\alpha_{j}\right)$ converges to the Dirac distribution in $\mathscr{Q}^{\prime}\left(\mathbf{R}^{n}\right)$. The main step is to show that $\tilde{F}\left(\alpha_{j}\right)$ converges in $\mathscr{D}^{\prime}\left(\mathbf{R}^{n}\right)$. We first outline the proof by the following four steps.

(i) Writing the integral for $\alpha_{j} * \psi$ as a limit of Riemann sums, the continuity of $\tilde{F}$ and property (3) imply

$$
\tilde{F}\left(\alpha_{j} * \psi\right)=(2 \pi)^{n / 2} \cdot \hat{\psi} \cdot \tilde{F}\left(\alpha_{j}\right)
$$

(ii) Since $\alpha_{j} * \psi \rightarrow \psi$ in $\mathscr{D}\left(\mathbf{R}^{n}\right)$, we obtain from (i) that in $\mathscr{Q}^{\prime}\left(\mathbf{R}^{n}\right)$

$$
(2 \pi)^{n / 2} \cdot \hat{\psi} \cdot \tilde{F}\left(\alpha_{j}\right) \rightarrow \tilde{F}(\psi) .
$$

(iii) Dividing in (ii) by $\hat{\psi}$, it follows that $\tilde{F}\left(\alpha_{j}\right) \rightarrow S$ in $\mathscr{D}^{\prime}\left(\mathbf{R}^{n}\right)$ and for each $\psi \in \mathscr{Q}\left(\mathbf{R}^{n}\right)$

$$
(2 \pi)^{n / 2} \cdot \hat{\psi} \cdot S=\tilde{F}(\psi)
$$


(iv) Properties (1) and (2) now imply $S$ is radial and homgeneous of degree 0 and therefore necessarily constant.

Finally (iii) gives that $\tilde{F}$ is a constant multiple of the Fourier transform. We now give the arguments to each step.

ad (i) Expanding $\alpha_{j} * \psi$ as a limit in $\mathscr{D}\left(\mathbf{R}^{n}\right)$ of Riemann sums, we obtain from the continuity of $\tilde{F}$ and property (3),

$$
\begin{aligned}
\tilde{F}\left(\alpha_{j} * \psi\right) & =\tilde{F}\left(\mathscr{D}-\lim _{\Delta t \rightarrow 0} \sum_{m} \alpha_{j}\left(\cdot-t_{m}\right) \psi\left(t_{m}\right) \Delta t\right) \\
& =\mathscr{Q}^{\prime}-\lim _{\Delta t \rightarrow 0}\left(\sum_{m} \tilde{F}\left(\alpha_{j}\left(\cdot-t_{m}\right)\right) \psi\left(t_{m}\right) \Delta t\right) \\
& =\mathscr{Q}^{\prime}-\lim _{\Delta t \rightarrow 0}\left(\sum_{m} \psi\left(t_{m}\right) e^{-i\left\langle\cdot t_{m}\right\rangle} \Delta t\right) \cdot \tilde{F}\left(\alpha_{j}\right) \\
& =(2 \pi)^{n / 2} \cdot \hat{\psi} \cdot \tilde{F}\left(\alpha_{j}\right) .
\end{aligned}
$$

To see that the last equality holds, we have to certify that for all $\theta \in \mathscr{D}\left(\mathbf{R}^{n}\right)$,

$$
\text { D }-\lim _{\Delta t \rightarrow 0} \theta\left(\sum_{m} \psi\left(t_{m}\right) e^{-i\left\langle\cdot, t_{m}\right\rangle} \Delta t\right)=(2 \pi)^{n / 2} \theta \cdot \hat{\psi} \text {. }
$$

Note that $H_{\Delta t}=\left\{\sum_{m} \psi\left(t_{m}\right) e^{-i\left\langle\cdot, t_{m}\right\rangle} \Delta t\right\}$ is a family of holomorphic functions on $\mathbf{C}^{n}$, pointwise convergent to $\hat{\psi}$. If $K$ denotes the compact support of $\psi$, we have for $z \in \mathbf{C}^{n}$,

$$
\left|\sum_{m} \psi\left(t_{m}\right) e^{-i\left\langle z, t_{m}\right\rangle} \Delta t\right| \leqslant\|\psi\|_{\infty} \cdot|K| \cdot e^{|\operatorname{Im}(z)|} .
$$

Hence $H_{\Delta t}$ is uniformly bounded in a complex neighbourhood of $\mathbf{R}^{n}$, i.e. $H_{\Delta t}$ is normal. From Vitali's theorem in several variables follows that $H_{\Delta t}$ converges uniformly in all derivatives on compact subsets of $\mathbf{R}^{n}$. Thus $\theta \cdot H_{\Delta t}$ converges in $\mathscr{D}\left(\mathbf{R}^{n}\right)$ against $\boldsymbol{\theta} \cdot \hat{\psi}$. Step (ii) is now an immediate consequence.

ad (iii) For any bounded open set $\Omega$ we may choose $\psi \in \mathscr{D}\left(\mathbf{R}^{n}\right)$ such that $\hat{\psi} \neq 0$ on $\Omega$. Dividing by $\hat{\psi}$, it follows that $\tilde{F}\left(\alpha_{j}\right)$ converges in $\mathscr{D}^{\prime}(\Omega)$. But a distribution is determined by its local behaviour, hence $\tilde{F}\left(\alpha_{j}\right)$ converges to a certain $S \in G D^{\prime}\left(\mathbf{R}^{n}\right)$.

ad (iv) Since the $\alpha_{j}$ are radial it is easy to check that property (1) implies $S$ is rotation invariant. In the same way, property (2) implies $S$ is homogeneous of degree 0 . But it is well known that such a distribution must be constant (this follows from Euler's equation for $S$ and the structure of point-supported distributions, see also Donoghue [2]).

The proof of the theorem is now complete.

The Fourier transform generally maps functions not into ' $\mathcal{D}^{\prime}$, but into $\mathscr{Z}^{\prime}$. Thus $\mathscr{Z}^{\prime}$ is the maximal space in which Fourier transforms of (locally integrable) functions may occur. Therefore, it may be more appropriate to consider for the operator $\tilde{F}$ in Theorem 1 the image space $\mathscr{Z}^{\prime}$ rather than $\mathscr{D}^{\prime}$. We now show that in this case the theorem holds also. 
CoRollaRY 1.1. Let $\tilde{F}: \mathscr{D}\left(\mathbf{R}^{n}\right) \rightarrow \mathcal{I}^{\prime}\left(\mathbf{R}^{n}\right)$ be a continuous operator. Then $\tilde{F}$ is a constant multiple of the Fourier transform, if and only if $\tilde{F}$ satisfies properties (1)-(3) of Theorem 1.

Proof. We only sketch the proof, which can be done by the same method as in Theorem 1. Instead of $\tilde{F}$, we consider the operator $T=F^{-1} \circ \tilde{F}$ from $\mathscr{D}\left(\mathbf{R}^{n}\right)$ into $D^{\prime}\left(\mathbf{R}^{n}\right)$, which is a continuous rotation, dilation, and translation invariant operator. We have to show that $T$ is a constant multiple of the identity.

By the analogous equation to (1.1), we conclude that

$$
T\left(\alpha_{j} * \psi\right)=T\left(\alpha_{j}\right) * \psi,
$$

which implies the existence of a distribution $M$, such that

$$
M=\lim _{j \rightarrow \infty} T\left(\alpha_{j}\right) \text { and } T \psi=M * \psi,
$$

(cf. Schwartz [13, Theorème XXIII]). In the same way as in the proof of Theorem 1, we verify that $M$ is rotation invariant and homogeneous of degree $-n$. But it is well known that if $M$ is a radial homogeneous distribution of degree $-n$ on $\mathbf{R}^{n}$, then $M=A \cdot \delta(x)$ for some constant $A$ (follows from Gelfand and Shilov [4] or Gårding [3]). Thus we have obtained $T \psi=A \psi$, and the corollary is proved.

CoRollaRy 1.2. Let $\tilde{F}: \mathscr{Z}\left(\mathbf{R}^{n}\right) \rightarrow \mathscr{D}^{\prime}\left(\mathbf{R}^{n}\right)$ respectively $\tilde{F}: \mathscr{Z}\left(\mathbf{R}^{n}\right) \rightarrow \mathscr{Z}^{\prime}\left(\mathbf{R}^{n}\right)$ be continuous operators. Then $\tilde{F}$ is a constant multiple of the Fourier transform, if and only if $\tilde{F}$ satisfies (1)-(3) of Theorem 1.

Proof. The first case of this Corollary ( $\tilde{F}$ from $\mathscr{Z}^{2}$ into $\left.\mathscr{D}^{\prime}\right)$ can be reduced to Corollary 1.1 in the following way. Instead of $\tilde{F}$, we consider its adjoint $\tilde{F}^{t}$ from $\mathscr{D}$ into $\mathscr{Z}^{\prime}$, defined by $(\psi \in \mathscr{D}, \theta \in \mathscr{Z})$

$$
\left\langle\tilde{F}^{t}(\psi), \theta\right\rangle=\langle\tilde{F} \theta, \psi\rangle .
$$

Then $\tilde{F}^{t}$ is a continuous operator, and it is easy to check that $\tilde{F}^{t}$ satisfies (1)-(3) also. Thus, if follows from Corollary 1.1 that $\tilde{F}^{t}$ is a constant multiple of the Fourier transform, and hence by (1.2) also is $\tilde{F}$.

On the other hand, the second case of this corollary ( $\tilde{F}$ from $\mathscr{Z}$ into $\left.\mathscr{Z}^{\prime}\right)$ can be reduced to Theorem 1 as follows. Instead of $\tilde{F}$, we consider the operator $T=$ $F \circ \tilde{F} \circ F^{-1}$ from $\mathscr{D}$ into $\mathscr{Q}^{\prime}$. Then $T$ is a continuous operator satisfying (1)-(3). Therefore $T$ is a constant multiple of the Fourier transform, and hence also is $\tilde{F}$.

RemarK 1.1. Theorem 1 applies also if $\tilde{F}: A \rightarrow B$ is a continuous operator, where $A$ and $B$ are spaces continuously embedded between $\mathscr{D}$ and $\mathscr{D}^{\prime}, \mathscr{D} \subset A, B \subset \mathscr{Q}^{\prime}$ (or between any pair of spaces occuring in Corollaries 1.1 and 1.2). Therefore, Theorem 1 applies to all kinds of $C^{k}, L^{p}$, Sobolev and Besov spaces. Hence Theorem 1 contains results of Cooper [1, Theorems 2 and 4] and Kober [10, Theorem 6']. Cooper and Kober characterized by different methods the Fourier transform in the case $n=1, A=L^{p}, B=L^{p \prime}, 1 \leqslant p \leqslant 2$, and further conditions on the image space $B$.

REMARK 1.2. Theorem 1 can be used to derive similar characterizations for transforms related to the Fourier transform. As an example, we consider the Hilbert 
transform $H$ on $L^{2}(\mathbf{R})$, given by

$$
(H f)^{\wedge}(x)=i \operatorname{sign}(x) \hat{f}(x) .
$$

If we now look for a continuous operator $\tilde{H}$ on $L^{2}(\mathbf{R})$, commuting with translations and dilations and anticommuting with reflection, we define $\tilde{F}: L^{2} \rightarrow L^{2}$ by

$$
(\tilde{F} f)(x)=-i \operatorname{sign}(x)(\tilde{H} f)^{\wedge}(x) .
$$

Clearly, $\tilde{F}$ satisfies (1)-(3) of Theorem 1. Thus, we obtain the known fact that $\tilde{H}$ must be a constant multiple of the Hilbert transform (cf. Cooper [1], Kober [10], and Stein [15, p. 55]).

REMARK 1.3. Any one of the conditions (1)-(3) in Theorem 1 is essential.

Proof. We only consider the case $n=1$; for arbitrary $n$, we can define analogous counterexamples of $\tilde{F}$ not being a constant multiple of the Fourier transform. First, we define

$$
(\tilde{F} \psi)(x)=\operatorname{sign}(x) \hat{\psi}(x) .
$$

Then $\tilde{F}$ satisfies (2) and (3), but not (1). Now, we define

$$
(\tilde{F} \psi)(x)=x^{2} \cdot \hat{\psi}(x)
$$

Then $\tilde{F}$ satisfies (1) and (3), but not (2). Finally, we define

$$
\tilde{F} \psi=L \psi,
$$

where $L$ is the Laplace transform. Then $\tilde{F}$ satisfies (1) and (2), but not (3).

In spite of Remark 1.3 we can omit the rotation property (1) in Theorem 1, if the range of $\tilde{F}$ consists of continuous functions (we equip the space $C\left(\mathbf{R}^{n}\right)$ of continuous functions on $\mathbf{R}^{n}$ with the topology induced by $\mathscr{D}^{\prime}\left(\mathbf{R}^{n}\right)$ ). This will be a consequence of the following corollary to the proof of Theorem 1, characterizing all continuous operators $\tilde{F}$ from $\mathscr{D}$ into $\mathscr{D}^{\prime}$ having the dilation and translation property of the Fourier transform.

CoROllaRY 1.3. Let $\tilde{F}: \mathscr{D}\left(\mathbf{R}^{n}\right) \rightarrow \mathscr{D}^{\prime}\left(\mathbf{R}^{n}\right)$ be a continuous operator. Then $\tilde{F}$ is a solution of the functional equations (2) and (3) in Theorem 1, if and only if $\tilde{F}$ has the form $\tilde{F}(\psi)=\hat{\psi} \cdot S$, where $S$ is a homogeneous distribution of degree 0 , i.e. $S$ satisfies Euler's equation

$$
\sum_{j=1}^{n} x_{j} \cdot \frac{\partial S}{\partial x_{j}}=0 .
$$

COROLlaRY 1.4. Let $\tilde{F}: \mathscr{D}\left(\mathbf{R}^{n}\right) \rightarrow C\left(\mathbf{R}^{n}\right)$ be a continuous operator, satisfying only conditions (2) and (3) of Theorem 1. Then $\tilde{F}$ is a constant multiple of the Fourier transform.

Proof. From Corollary 1.3 we know that $\tilde{F}(\psi)=\hat{\psi} \cdot S$ for each $\psi \in \mathscr{Q}\left(\mathbf{R}^{n}\right)$, where $S$ is homogeneous of degree 0 . But the continuity of each $\tilde{F}(\psi)$ implies the continuity of $S$ and therefore $S$ must be constant. Indeed, this argument shows that only continuity at 0 of the functions in the range of $\tilde{F}$ is needed.

Moreover, Remark 1.3 shows that this corollary fails, i.e. the rotation property cannot be omitted, if the functions in the range of $\tilde{F}$ are not continuous at 0 . 
REMARK 1.4. The proof of Theorem 1 and Corollary 1.3 connect our results to articles of Hardy [7] and Plancherel [12], who (by different methods) looked on $L^{2}(\mathbf{R})$ for solutions of equation (2) in Theorem 1 in order to find out "all" Fourier kernels (kernels which give rise to an involutionary integral transform).

2. A characterization of the Radon transform. In this section, we first show by a counterexample that the Radon transform is not determined up to a constant multiple as an operator from $\mathscr{D}\left(\mathbf{R}^{n}\right)$ into $\mathscr{D}^{\prime}\left(S^{n-1} \times \mathbf{R}\right)$ by its behaviour under rotations, dilations, and translations. This is a contrast to the result in the case of the Fourier transform (Theorem 1). But we are able to characterize the Radon transform by restricting the range space to integrable distributions. Because the range of the Radon transform consists of functions on the product space $S^{n-1} \times \mathbf{R}$, it is convenient to regard the range space as a tensor product space. In fact, we characterize the Radon transform as an operator from $\mathscr{Q}\left(\mathbf{R}^{n}\right)$ into $\mathscr{D}_{L^{\prime}}^{\prime}\left(S^{n-1} \times \mathbf{R}\right)$, and in view of Proposition 2.1 this is in some sense the best possible result.

The Radon transform $R$ is a continuous operator from $L^{1}\left(\mathbf{R}^{n}\right)$ into $L^{1}\left(S^{n-1} \times \mathbf{R}\right)$, defined by

$$
(R f)(\omega, p)=\int_{\langle\omega, y\rangle=p} f(y) d y,
$$

where we equip $S^{n-1} \times \mathbf{R}$ with the product of the Euclidean surface measure on $S^{n-1}$ and the Lebesgue measure on $\mathbf{R}$. The Radon transform is injective, and hence inverting $R$ means reconstructing a function from its integrals along hyperplanes. The Radon and Fourier transforms are related as follows (see Gelfand, Graev, and Vilenkin [5]).

$$
(F f)(p \omega)=(2 \pi)^{-n / 2} \int_{\mathbf{R}} e^{-i s p}(R f)(\omega, s) d s .
$$

It is well known that the Radon transform has the properties $\left(0.1^{\prime}\right)-\left(0.3^{\prime}\right)$ in the introduction (see again [5]). We now state the announced counterexample to the characterization of the Radon transform.

Proposition 2.1. There exist two linearly independent continuous operators $R_{1}$ and $R_{2}$,

$$
R_{1}, R_{2}: \mathscr{D}\left(\mathbf{R}^{n}\right) \rightarrow L^{2}\left(S^{n-1} \times \mathbf{R}\right),
$$

which both satisfy the conditions $\left(0.1^{\prime}\right)-\left(0.3^{\prime}\right)$ in the introduction.

Proof. Of course, we can choose $R_{1}$ to be the Radon transform. We now define a linear operator $R_{2}$ on $\mathscr{D}\left(\mathbf{R}^{n}\right)$ by

$$
\left(R_{2} \psi\right)(\omega, p)=\int_{\mathbf{R}} \operatorname{sign}(s) \hat{\psi}(s \omega) e^{i p s} d s .
$$

First, it follows from relation (2.1) that the Radon transform and $R_{2}$ are linearly independent. Now let $\left(\psi_{k}\right)$ be a sequence converging to 0 in $\mathscr{D}\left(\mathbf{R}^{n}\right)$. Then the functions

$$
\theta_{k, \omega}(s)=\operatorname{sign}(s) \hat{\psi}_{k}(s \omega)
$$


converge to 0 in $L^{2}(\mathbf{R})$ uniformly in $\omega$, because

$$
\begin{aligned}
\left\|\theta_{k, \omega}\right\|_{2}^{2} & =\int_{\mathbf{R}}\left|\hat{\psi}_{k}(s \omega)\right|^{2} d s \\
& =(2 \pi)^{-1} \int_{\mathbf{R}}\left|\int_{\mathbf{R}} \hat{\psi}_{k}(p \omega) e^{i s p} d p\right|^{2} d s \\
& =(2 \pi)^{-1}(2 \pi)^{2-n} \int_{\mathbf{R}}\left|\left(R \psi_{k}\right)(\omega, s)\right|^{2} d s \\
& \leqslant(2 \pi)^{1-n} \cdot 2 r\left\|R \psi_{k}\right\|_{\infty}^{2} \\
& \leqslant 2(2 \pi)^{1-n} \cdot \gamma_{n-1}^{2}(r) \cdot r\left\|\psi_{k}\right\|_{\infty}^{2},
\end{aligned}
$$

where $r>0$ is such that all $\psi_{k}$ have support in $\{|x| \leqslant r\}$ and $\gamma_{n}(r)$ denotes the volume of $\{|x| \leqslant r\}$ in $\mathbf{R}^{n}$. Indeed, the second equation is Plancherel's identity on $\mathbf{R}$ applied to $\hat{\psi}_{k}(s \omega)$ for each $\omega$, and the third equation is derived from (2.1) by inverse Fourier transform. We obtain

$$
\begin{aligned}
\left\|R_{2} \psi\right\|_{2}^{2} & =\int_{S^{n-1}} \int_{\mathbf{R}}\left|\int_{\mathbf{R}} \theta_{k, \omega}(s) e^{i p s} d s\right|^{2} d p d \omega \\
& =2 \pi \int_{S^{n-1}}\left\|\hat{\theta}_{k, \omega}\right\|_{2}^{2} d \omega \\
& =2 \pi \int_{S^{n-1}}\left\|\theta_{k, \omega}\right\|_{2}^{2} d \omega \\
& =4 \pi(2 \pi)^{1-n} \cdot \omega_{n} \cdot \gamma_{n-1}^{2}(r) \cdot r\left\|\psi_{k}\right\|_{\infty}^{2},
\end{aligned}
$$

where $\omega_{n}$ is the Euclidean surface measure of $S^{n-1}$. Thus $R_{2}$ is a continuous operator into $L^{2}\left(S^{n-1} \times \mathbf{R}\right)$. Further, it is easily checked that $R_{2}$ satisfies conditions $\left(0.1^{\prime}\right)-$ $\left(0.3^{\prime}\right)$.

Moreover, this example of $R_{2}$ shows that in the proposition we could even take functions which are $C^{\infty}$ in $\omega$ on $S^{n-1}$ as image space of $R_{1}$ and $R_{2}$. The proof of the proposition is complete.

In order to characterize the Radon transform, Proposition 2.1 shows that one has to restrict the range space. Indeed, we need a condition on the second component for distributions on $S^{n-1} \times \mathbf{R}$. Observing that $\mathscr{D}^{\prime}\left(S^{n-1} \times \mathbf{R}\right)=\mathscr{Q}^{\prime}\left(S^{n-1}\right) \hat{\otimes}_{\pi} \mathscr{D}^{\prime}(\mathbf{R})$, it is thus convenient to use tensor products. To this purpose, we define the space $\mathscr{D}_{L^{\prime}}^{\prime}(\mathbf{R})$ of integrable distributions, following Schwartz [13, Chapter VI, §8].

We denote by $C_{0}^{\infty}(\mathbf{R})$ the space of all $C^{\infty}$ functions on $\mathbf{R}$, tending to 0 at infinity together with all their derivatives. The topology of $C_{0}^{\infty}$ is given by the following sequence $\left(p_{k}\right)$ of norms.

$$
p_{k}(\psi)=\max _{m \leqslant k} \sup _{x}\left|D^{m} \psi(x)\right| .
$$

By $\mathscr{D}_{L^{1}}^{\prime}(\mathbf{R})$ we denote the topological dual of $C_{0}^{\infty}(\mathbf{R})$. In just the same manner we define the spaces $C_{0}^{\infty}\left(S^{n-1} \times \mathbf{R}\right)$ and $\mathscr{D}_{L^{\prime}}^{\prime}\left(S^{n-1} \times \mathbf{R}\right)$, the latter being the integrable distributions on $S^{n-1} \times \mathbf{R}$. For technical reasons, we rather deal with the space $\mathscr{D}^{\prime}\left(S^{n-1}\right) \hat{\otimes}_{\pi} \mathscr{D}_{L^{1}}^{\prime}(\mathbf{R})$, the completion of the $\pi$-tensor product of $\mathscr{Q}^{\prime}\left(S^{n-1}\right)$ and 
$\mathscr{Q}_{L^{1}}^{\prime}(\mathbf{R})$, where both factors are complete (DF)-spaces and $\mathscr{D}^{\prime}\left(S^{n-1}\right)$ is nuclear. In fact, the "integrable kernel theorem" of Schwartz [14, Exposé 21] yields

$$
\mathscr{D}_{L^{1}}^{\prime}\left(S^{n-1} \times \mathbf{R}\right)=\mathscr{D}^{\prime}\left(S^{n-1}\right) \hat{\otimes}_{\pi} \mathscr{D}_{L^{\prime}}^{\prime}(\mathbf{R}) \text {. }
$$

Thus this tensor product space is a subspace of $\mathscr{D}^{\prime}\left(S^{n-1} \times \mathbf{R}\right)$, locally containing all distributions and globally all distributions with compact support as well as all finite measures on $S^{n-1} \times \mathbf{R}$, and hence containing the range of the Radon transform. Within this setup, we now are able to characterize the Radon transform.

THEOREM 2. Let $\tilde{R}: \mathscr{D}\left(\mathbf{R}^{n}\right) \rightarrow \mathscr{Q}_{L^{1}}^{\prime}\left(S^{n-1} \times \mathbf{R}\right)$ be a continuous operator. Then $\tilde{R}$ is a constant multiple of the Radon transform, if and only if

$\left(1^{\prime}\right)\left(\tilde{R} \psi_{U}\right)(\omega, p)=(\tilde{R} \psi)(U \omega, p)$,

$\left(2^{\prime}\right)\left(\tilde{R} \psi_{\delta}\right)(\omega, p)=\delta^{1-n}(\tilde{R} \psi)(\omega, \delta p)$,

(3') $\left(\tilde{R} \psi_{a}\right)(\omega, p)=(\tilde{R} \psi)(\omega, p+\langle\omega, a\rangle)$,

where $\psi \in \mathscr{Q}\left(\mathbf{R}^{n}\right), U \in O(n), \delta>0$, and $a \in \mathbf{R}^{n}$.

Proof. We assume that $\tilde{R}$ has properties $\left(1^{\prime}\right)-\left(3^{\prime}\right)$. Let $\alpha \in \mathscr{D}\left(\mathbf{R}^{n}\right)$ be rotation invariant. Then, if $U$ is any rotation, we know from $\left(1^{\prime}\right)$

$$
\tilde{R} \alpha(\omega, p)=\tilde{R} \alpha(U \omega, p) .
$$

We first show that there exists $T \in \mathscr{Q}_{L^{\prime}}^{\prime}(\mathbf{R})$, such that $\tilde{R} \alpha(\omega, p)=T(p)$, i.e.

$$
\langle\tilde{R} \alpha(\omega, p), \theta(\omega, p)\rangle=\int_{S^{n-1}}\langle T(p), \theta(\omega, p)\rangle d \omega
$$

for all $\theta \in \mathscr{D}\left(S^{n-1} \times \mathbf{R}\right)$. We define $T$ by

$$
\langle T(p), \psi(p)\rangle=\omega_{n}^{-1} \cdot\langle\tilde{R} \alpha(\omega, p), 1(\omega) \psi(p)\rangle .
$$

Obviously $T \in \mathscr{Q}^{\prime}(\mathbf{R})$, and we want to show that $T \in \mathscr{D}_{L^{1}}^{\prime}(\mathbf{R})$. Now, since both $\mathscr{D}^{\prime}\left(S^{n-1}\right)$ and $\mathscr{D}_{L^{\prime}}^{\prime}(\mathbf{R})$ are complete (DF)-spaces and $\mathscr{D}^{\prime}\left(S^{n-1}\right)$ is nuclear, we have the following representation of the $\pi$-tensor product (cf. Grothendieck [6, Chapter II, §3.1]).

$$
\tilde{R} \alpha=\sum_{j=1}^{\infty} \lambda_{j} S_{j} \otimes T_{j},
$$

where $\left(S_{j}\right)$ and $\left(T_{j}\right)$ are bounded sequences in $\mathscr{Q}^{\prime}\left(S^{n-1}\right)$ and $\mathscr{D}_{L^{\prime}}^{\prime}(\mathbf{R})$ respectively, and $\left(\lambda_{j}\right)$ is an absolutely summable sequence. We obtain

$$
\langle\tilde{R} \alpha(\omega, p), 1(\omega) \psi(p)\rangle=\sum_{j=1}^{\infty} \lambda_{j}\left\langle S_{j}, 1\right\rangle\left\langle T_{j}, \psi\right\rangle .
$$

Defining $\tilde{\lambda}_{j}=\lambda_{j}\left\langle S_{j}, 1\right\rangle$, the boundedness of $\left(S_{j}\right)$ implies the absolute summability of $\left(\tilde{\lambda}_{j}\right)$. Hence we have

$$
T=\omega_{n}^{-1} \cdot \sum_{j=1}^{\infty} \tilde{\lambda}_{j} T_{j}
$$

which means that $T$ is an element of $\mathscr{D}_{L^{1}}^{\prime}(\mathbf{R})$ having the required property $T=\tilde{R} \alpha$ above, as is easily seen. Moreover, the tensor representation implies that $\int T d p=$ $\iint \tilde{R} \alpha d p d \omega$. Thus, in the following we simply write

$$
\tilde{R} \alpha(\omega, p)=T(p)=\tilde{R} \alpha(p) .
$$


Next we verify the following relation if $T \in \mathscr{Q}^{\prime}(\mathbf{R})$ and $\psi, \theta \in \mathscr{Q}(\mathbf{R})$.

$$
\int_{\mathbf{R}} \psi(q)\langle T(p), \theta(p+q)\rangle d q=\int_{\mathbf{R}} \theta(q)\langle T(p), \psi(p-q)\rangle d q .
$$

Indeed, we have

$$
\begin{aligned}
\int_{\mathbf{R}} \psi(q)\langle T(p), \theta(p+q)\rangle d q & =\int_{\mathbf{R}} \psi(-q)\langle T(p), \theta(p-q)\rangle d q \\
& =(T * \tilde{\theta}) * \psi(0),
\end{aligned}
$$

where $\tilde{\theta}(p)=\theta(-p)$. On the other hand

$$
\int_{\mathbf{R}} \theta(q)\langle T(p), \psi(q-p)\rangle d q=(T * \psi) * \tilde{\theta}(0) .
$$

Since $(T * \tilde{\theta}) * \psi=(T * \psi) * \tilde{\theta}$, relation (2.2) follows.

Let now $\alpha, \psi \in \mathscr{D}\left(\mathbf{R}^{n}\right)$ and $\alpha$ again be radial. Expanding the convolution $\psi * \alpha$ in its Riemann sums, we obtain by using property $\left(3^{\prime}\right)$

$$
\tilde{R}(\psi * \alpha)(\omega, p)=\lim _{\Delta t \rightarrow 0} \sum_{m} \psi\left(t_{m}\right) \tilde{R} \alpha\left(p-\left\langle\omega, t_{m}\right\rangle\right) \Delta t
$$

Thus for $\theta \in \mathscr{Q}\left(S^{n-1} \times \mathbf{R}\right)$ we have

$$
\begin{aligned}
\langle\tilde{R}(\psi * \alpha)(\omega, p), \theta(\omega, p)\rangle & \\
& =\lim _{\Delta t \rightarrow 0} \sum_{m} \psi\left(t_{m}\right) \int_{S^{n-1}}\left\langle\tilde{R} \alpha(p), \theta\left(\omega, p+\left\langle\omega, t_{m}\right\rangle\right)\right\rangle d \omega \Delta t \\
& =\int_{\mathbf{R}^{n}} \int_{S^{n-1}} \psi(t)\langle\tilde{R} \alpha(p), \theta(\omega, p+\langle\omega, t\rangle)\rangle d \omega d t \\
& =\int_{S^{n-1}} \int_{\mathbf{R}^{n}} \psi(t)\langle\tilde{R} \alpha(p), \theta(\omega, p+\langle\omega, t\rangle)\rangle d t d \omega \\
& =\int_{S^{n-1}} \int_{\mathbf{R}}\langle\tilde{R} \alpha(p), R \psi(\omega, q-p)\rangle \theta(\omega, q) d q d \omega
\end{aligned}
$$

where the last equality follows from (2.2) and the definition of the Radon transform. Now let $\left(\alpha_{j}\right)$ be a regularization in $\mathscr{D}\left(\mathbf{R}^{n}\right)$, derived from a radial $\alpha \in \mathscr{D}\left(\mathbf{R}^{n}\right)$. Observing $\alpha_{j}(x)=j^{n} \alpha(j x), j \geqslant 1$, we obtain by property (2')

$$
\begin{aligned}
\left\langle\tilde{R}\left(\psi * \alpha_{j}\right)(\omega, p), \theta(\omega, p)\right\rangle & =j \int_{S^{n-1}} \int_{\mathbf{R}}\langle\tilde{R} \alpha(j p), R \psi(\omega, q-p)\rangle \theta(\omega, p) d q d \omega \\
& =\int_{S^{n-1}} \int_{\mathbf{R}}\left\langle\tilde{R} \alpha(p), R \psi\left(\omega, q-\frac{p}{j}\right)\right\rangle \theta(\omega, q) d q d \omega .
\end{aligned}
$$

Now, for all (fixed) $\omega_{0}$ and $q_{0}$, the sequence of functions $R \psi\left(\omega_{0}, q_{0}-p / j\right)$ is an approximation in $C_{0}^{\infty}(\mathbf{R})$ of the constant function $R \psi\left(\omega_{0}, q_{0}\right)$, because this sequence converges to $R \psi\left(\omega_{0}, q_{0}\right)$ in $C^{\infty}(\mathbf{R})$ and is bounded with respect to the norms $p_{k}$, $k \geqslant 1$. In fact, for all $m$ there exists a constant $A$, such that for all $j$

$$
\left|D^{m}(R \psi)\left(\omega_{0}, q_{0}-p / j\right)\right| \leqslant A \text {. }
$$


But since $\tilde{R} \alpha \in \mathscr{D}_{L^{\prime}}^{\prime}(\mathbf{R})$, it follows that the limit

$$
\lim _{j \rightarrow \infty}\left\langle\tilde{R} \alpha(p), R \psi\left(\omega_{0}, q_{0}-p / j\right)\right\rangle
$$

exists (see Schwartz [13, p. 203]). Hence we obtain

$$
\begin{aligned}
\langle\tilde{R} \psi, \theta\rangle & =\left\langle\tilde{R}\left(\lim _{j \rightarrow \infty} \psi * \alpha_{j}\right), \theta\right\rangle=\lim _{j \rightarrow \infty}\left\langle\tilde{R}\left(\psi * \alpha_{j}\right), \theta\right\rangle \\
& =\lim _{j \rightarrow \infty} \int_{S^{n-1}} \int_{\mathbf{R}}\left\langle\tilde{R} \alpha(p), R \psi\left(\omega, q-\frac{p}{j}\right)\right\rangle \theta(\omega, q) d q d \omega \\
& =\langle\tilde{R} \alpha, 1\rangle \int_{S^{n-1}} \int_{\mathbf{R}} R \psi(\omega, q) \theta(\omega, q) d q d \omega=\langle\tilde{R} \alpha, 1\rangle \cdot\langle R \psi, \theta\rangle,
\end{aligned}
$$

where equation (2.3) follows by Lebesgue's convergence theorem. Summarizing, we have shown that

$$
\tilde{R} \psi=\langle\tilde{R} \alpha, 1\rangle \cdot R \psi
$$

and the theorem is proved.

REMARK 2.1. Any one of the conditions $\left(1^{\prime}\right)-\left(3^{\prime}\right)$ in Theorem 2 is essential. This can be seen by analogous counterexamples as given in Remark 1.3, by using relation (2.1) between the Fourier and Radon transforms (or directly by analyzing the proof of Theorem 2).

Further, the following may be interesting. In contrast to the case of the Fourier transform (Corollary 1.4 ), we cannot omit the rotation property $\left(1^{\prime}\right)$ by restricting the range. In fact, there exists $\tilde{R}: \mathscr{D}\left(\mathbf{R}^{n}\right) \rightarrow \mathscr{D}\left(S^{n-1} \times \mathbf{R}\right)$, not being a constant multiple of the Radon transform, but satisfying $\left(2^{\prime}\right)$ and $\left(3^{\prime}\right)$.

Finally, we discuss a possibility to modify Theorem 2 . In view of the counterexample in Proposition 2.1 it seems to be difficult to enlarge the range space even slightly. But, imposing further conditions on the behaviour of $\tilde{R}$, namely considering also negative dilations, and using the characterization of the Fourier transform (Theorem $1)$, we can prove Theorem 2 in the case of the range space $L^{p}\left(S^{n-1} \times \mathbf{R}\right), 1 \leqslant p \leqslant 2$.

COROllaRY 2.1. Let $\tilde{R}: \mathscr{D}\left(\mathbf{R}^{n}\right) \rightarrow L^{p}\left(S^{n-1} \times \mathbf{R}\right), 1 \leqslant p \leqslant 2$, be a continuous operator satisfying conditions $\left(1^{\prime}\right)$ and $\left(3^{\prime}\right)$ of Theorem 2 , and further the condition

$\left(2^{\prime \prime}\right)\left(\tilde{R} \psi_{\delta}\right)(\omega, p)=|\delta|^{1-n}(\tilde{R} \psi)(\omega, \delta p)$ for all dilations $\delta \neq 0$. Then $\tilde{R}$ is a constant multiple of the Radon transform.

Proof. Let $\tilde{R}$ be an operator satisfying the assumptions of the corollary. We first observe that $\tilde{R}$ is even, i.e. $\tilde{R} \psi(\omega, p)=\tilde{R} \psi(-\omega,-p)$. In fact, from properties $\left(1^{\prime}\right)$ and $\left(2^{\prime \prime}\right)$ we obtain

$$
\tilde{R} \psi(-\omega,-p)=\tilde{R} \tilde{\psi}(\omega,-p)=\tilde{R} \psi(\omega, p),
$$

where $\tilde{\psi}$ denotes the function $\tilde{\psi}(x)=\psi(-x)$. In order to apply Theorem 1 , we define an operator $\tilde{F}$ from $\mathscr{Q}\left(\mathbf{R}^{n}\right)$ into $\mathscr{Q}{ }^{\prime}\left(\mathbf{R}^{n}\right)$ as follows.

$$
(\tilde{F} \psi)(p \omega)=\int_{\mathbf{R}} e^{-i s p}(\tilde{R} \psi)(\omega, s) d s,
$$


where $p \in \mathbf{R}$ and $\omega \in S^{n-1}$. Note that $\tilde{F} \psi$ is well defined almost everywhere on $\mathbf{R}^{n}$ since $\tilde{R}$ is even. Further, $\tilde{R} \psi(\omega, \cdot)^{\wedge}$ belongs to $L^{q}(\mathbf{R})$, where $\frac{1}{p}+\frac{1}{q}=1$, for almost all $\omega \in S^{n-1}$; this follows by Titchmarch's theorem (the Fourier transform maps $L^{p}$ into $L^{q}$ if $1 \leqslant p \leqslant 2$, cf. Donoghue [2, §51]). Now the following chain of inequalities shows that $\tilde{F}$ is continuous from $\mathscr{D}$ into $\mathscr{Q}^{\prime}$ (let $r>0, \psi \in \mathscr{Q}\left(\mathbf{R}^{n}\right)$ and let $F_{t}$ denote the Fourier transform with respect to the real variable $t$ ).

$$
\begin{aligned}
\int_{|x| \leqslant r}|\tilde{F} \psi(x)| d x=\int_{0}^{r} \int_{S^{n-1}}\left|\int_{\mathbf{R}} e^{-i s t}(\tilde{R} \psi)(\omega, s) d s\right| t^{n-1} d \omega d t \\
\quad=(2 \pi)^{1 / 2} \int_{S^{n-1}} \int_{0}^{r}\left|F_{t}(\tilde{R} \psi)(\omega, t)\right| t^{n-1} d t d \omega \\
\quad \leqslant(2 \pi)^{1 / 2} r^{n-1} \int_{S^{n-1}} \int_{0}^{r}\left|F_{t}(\tilde{R} \psi)(\omega, t)\right| d t d \omega \\
\leqslant(2 \pi)^{1 / 2} r^{n-1} \cdot r^{1-1 / q} \int_{S^{n-1}}\left(\int_{0}^{r}\left|F_{t}(\tilde{R} \psi)(\omega, t)\right|^{q} d t\right)^{1 / q} d \omega \\
\leqslant(2 \pi)^{1 / 2} r^{n-1 / q} \int_{S^{n-1}}\left(\int_{\mathbf{R}}\left|F_{t}(\tilde{R} \psi)(\omega, t)\right|^{q} d t\right)^{1 / q} d \omega \\
\leqslant(2 \pi)^{1 / q} \cdot r^{n-1 / q} \int_{S^{n-1}}\left(\int_{\mathbf{R}}|\tilde{R} \psi(\omega, s)|^{p} d s\right)^{1 / p} d \omega \\
=(2 \pi)^{1 / q} \cdot r^{n-1 / q} \cdot \omega_{n} \int_{S^{n-1}} \omega_{n}^{-1}\left(\int_{\mathbf{R}}|\tilde{R} \psi(\omega, s)|^{p} d s\right)^{1 / p} d \omega \\
\leqslant(2 \pi)^{1 / q} \cdot r^{n-1 / q} \cdot \omega_{n}^{1-1 / p}\left(\int_{S^{n-1}} \int_{\mathbf{R}}|\tilde{R} \psi(\omega, s)|^{p} d s d \omega\right)^{1 / p} \\
\leqslant(2 \pi)^{1 / q} \cdot r^{n-1 / q} \cdot \omega_{n}^{q} \cdot\|\tilde{R} \psi\|_{p},
\end{aligned}
$$

where inequality (2.4) follows from the Hausdorff-Young inequality for the onedimensional Fourier transform (cf. Donoghue [2, §51]), and inequality (2.5) follows from Jensen's inequality (cf. Jacobs $[9$, p. 116]) applied to the probability measure $\omega_{n}^{-1} d \omega$ and the convex function $-x^{1 / p}$. Hence $\tilde{F}$ is a continuous operator from $\mathscr{D}$ into $\mathscr{D}^{\prime}$. It is a straightforward computation to see that $\tilde{F}$ satisfies conditions (1)-(3) of Theorem 1. Thus $\tilde{F}$ is a constant multiple of the Fourier transform. Now, the definition of $\tilde{F}$ and relation (2.1) between the Radon and the Fourier transform show that for almost all $\omega \in S^{n-1}$ the function $\tilde{R} \psi(\omega, \cdot)$ is a constant multiple of $R \psi(\omega, \cdot)$, where the constant does not depend on $\psi$. This finishes the proof in the case $1<p \leqslant 2$. In the case $p=1$ (and $q=\infty$ ) some steps of the present proof are delicate. But this case $p=1$ is in fact contained in Theorem 2, observing that

$$
L^{1}\left(S^{n-1} \times \mathbf{R}\right)=L^{1}\left(S^{n-1}\right) \hat{\otimes}_{\pi} L^{1}(\mathbf{R})
$$

and that $L^{1}$ is contained in $\mathscr{D}_{L^{1}}^{\prime}$. Thus the proof of the corollary is complete. 


\section{REFERENCES}

1. J. L. B. Cooper, Functional equations for linear transformations, Proc. London Math. Soc. (3) 20 (1970), 1-32.

2. W. F. Dono ¿nue, Distributions and Fourier transforms, Academic Press, New York, 1969.

3. L. Gårdin ${ }_{\circ}$, :'ransformation de Fourier des distributions homogènes, Bull. Soc. Math. France 89 (1961), 381-428.

4. I. M. Gelfand and G. E. Shilov, Generalized functions. Vol. I, Academic Press, New York, 1964.

5. I. M. Gelfand, M. I. Graev and N. Ya. Vilenkin, Generalized functions. Vol. V, Academic Press, New York, 1966.

6. A. Grothendieck, Produits tensoriels topologiques et espaces nucléaires, Mem. Amer. Math. Soc. No. $16(1955)$.

7. G. H. Hardy, The resultant of two Fourier kernels, Proc. Cambridge Philos. Soc. 31 (1935), 1-6.

8. A. Hertle, Zur Radon-Transformation von Funktionen und Massen, Thesis, Erlangen, 1979.

9. K. Jacobs, Measure and integral, Academic Press, New York, 1978.

10. H. Kober, On functional equations and bounded linear transformations, Proc. London Math. Soc. (3) 14 (1964), 495-519.

11. D. Ludwig, The Radon transform on Euclidean space, Comm. Pure Appl. Math. 19 (1966), 49-81.

12. M. Plancherel, Quelques remarques à propos d'une note de G. H. Hardy: the resultant of two Fourier kernels, Proc. Cambridge Philos. Soc. 33 (1937), 413-418.

13. L. Schwartz, Théorie des distributions, Hermann, Paris, 1966.

14. __ Produits tensoriels topologiques d'espaces vectoriels topologiques, Séminaire Schwartz, Anneé 1953/1954, Paris 1954.

15. E. M. Stein, Singular integrals and differentiability properties of functions, Princeton Univ. Press, Princeton, N. J., 1970.

Fachbereich Mathematik, Universität Mainz, SaARstrasse 21, D-6500 Mainz, Federal RepubLIC OF GERMANY 